\title{
Le CNTF sauve les motoneurones chez la souris mutante pmn
}

Nous avions rapporté au début de cette année (voir $\mathrm{m} / \mathrm{s} n^{\circ} 2$, vol. 8, p. 176) la communication faite à la Society For Neuroscience par Ron Lindsay, des laboratoires Regeneron (USA), concernant les travaux de son équipe en vue de l'utilisation du CNTF (ciliary neurotrophic factor) comme agent thérapeutique dans les maladies motoneuronales. Aucune publication de ce groupe n'est venue confirmer jusqu'à présent, ni l'effet décrit chez la souris mutante $\mathrm{MnD}$ (motoneuronal degeneration), ni le démarrage des essais cliniques annoncés chez des patients atteints de sclérose latérale amyotrophique. C'est une autre équipe, animée par Michael Sendtner, Georg Kreutzberg et Hans Thoenen au Max-Planck de Martinsried (Allemagne), qui apporte aujourd'hui des éléments expérimentaux déterminants à l'appui de cet effet anti-neurodégénératif du CNTF [1].

L'équipe allemande a travaillé sur une autre souris mutante, la souris homozygote pmn/pmn (progressive motor neuronopathy) qui présente une atteinte motoneuronale, très proche de celle des amyotrophies spinales infantiles de type Werdnig Hoffmann, normalement létale en six à sept semaines en raison de troubles respiratoires [2]. Chez ces souris, l'apport continu de CNTF a déterminé une nette augmentation du temps de survie associée à une relative préservation - vérifiée histologiquement - des motoneurones et de leurs projections neuromusculaires. L'activité motrice des souris traitées était également nettement supérieure à celle des témoins. Il s'agit là de la première publication d'un traitement efficace contre une maladie motoneuronale et, comme nous l'avions indiqué il y a quelques mois, ces résultats off rent de grands espoirs pour les maladies humaines correspondantes. On peut noter que, si l'on met à part la publication de l'équipe de Matt Lavail sur l'effet du bFGF dans la rétinopathie pigmentaire d'origine génétique [3], il s'agit de la première démonstration de l'effet bénéfique d'un traitement par un facteur neurotrophique dans le système nerveux central.

Deux grandes questions restent posées dans le cadre d'une application clinique rapide de ces résultats. Tout d'abord, la que. Cette étude révèle incidemment (les auteurs ne le mentionnent que comme " résultats non publiés ") que la demi-vie du CNTF injecté par voie intra-veineuse ne serait que de quelques minutes. Afin de pallier ce problème, les auteurs ont implanté, dans la région péritonéale, des cellules de la lignée murine D3 dans lesquelles ils avaient introduit le gène codant pour le CNTF. La construction incluait également la séquence-signal du pré-pro NGF afin d'assurer la sécrétion du CNTF qui ne possède pas naturellement une telle séquence [4]. Cette implantation a assuré en continu un taux de facteur trophique sérique élevé auquel on peut sans doute attribuer l'effet thérapeutique. Il est difficile d'envisager, à l'heure actuelle, un tel mode d'administration chez les patients. On peut toutefois envisager d'autres voies telles que des mini-pompes implantables - qui auraient l'avantage de pouvoir libérer spécifiquement le facteur dans le système nerveux central - des transplants intracérébraux de polymères résorbables ou de fibroblastes génétiquement modifiés, etc. Le parcours vers des essais cliniques passe obligatoirement par des essais expérimentaux de ces divers modes d'administration afin de déterminer le plus adéquat, étant entendu qu'il ne s'agira plus là de maintenir des souris en vie pendant quelques semaines mais des hommes pour plusieurs dizaines d'années. Le second problème, pour lequel les éléments disponibles sont nettement plus douteux, est celui de l'effet d'une administration de CNTF à long terme sur le système nerveux, notamment au cours du développement. Dans le cas de la souris pmn, le traitement a été entrepris à la fin de la troisième semaine, lorsque l'homozygotie (et donc la pathologie) a été cliniquement reconnue. Une très grande part du développement du système nerveux central est alors accomplie chez la souris et on ne peut donc pas juger d'un effet du facteur sur le fonctionnement du système nerveux central (ce que le temps de survie expérimental - quelques semaines - ne permettait de toute façon pas). A ce moment, la maladie est déjà très avancée et le nombre de motoneurones que l'on peut sauver est réduit. Lorsque l'on s'intéressera directement au traitement d'amyotrophies spinales infantiles, un démarrage du traitement le plus précoce possible sera évidemment envisagé pour maintenir en vie un nombre de motoneurones aussi important que possible. L'apport massif d'un facteur trophique apparemment très puissant peut-il modifier l'architecture nerveuse et, éventuellement, induire des effets délétères majeurs? L'utilisation du CNTF dans les amyotrophies spinales infantiles passe, à l'évidence, par des études toxicologiques poussées chez le primate nonhumain en développement dans le but d'obtenir une réponse très précise à cette question.

Ce problème de toxicité potentielle au cours du développement est a prion moins crucial en ce qui concerne une décision d'application thérapeutique dans la sclérose latérale amyotrophique qui touche les adultes. Les résultats publiés ici sur la souris pmn ne contre-indiquent évidemment pas un tel essai thérapeutique. Le modèle expérimental pmn est, toutefois, très éloigné des anomalies observées dans la SLA et on aimerait, pour être convaincus de l'effet du CNTF sur les motoneurones à tous les stades de leur existence, voir enfin publiés les résultats chez $\mathrm{MnD}$ annoncés voilà déjà presque un an. Une nouvelle ère pourrait alors s'ouvrir enfin dans la thérapeutique neurologique, avec l'arrivée tant attendue de facteurs capables de retarder - voire d'arrêter - l'évolution des maladies neurodégénératives et non plus seulement d'en compenser certains effets.

M.P.

1. Sendtner M, Schmalbruch $\mathrm{H}$, Stöckli KA, $e$ al. Ciliary neutrophic factor prevents degeneration of motor neurons in mouse mutant progressive motor neuronopathy. Nature 1992: 358 : 502-4.

2. Schmalbruch $\mathrm{H}$, Jensen $\mathrm{HJ}$, Bjacrg $\mathrm{M}$, et al. A new mouse mutant with progressive motor neuropathy. J Neuropathol Exp Neurol $1991 ; 50$ : 192-204.

3. Faktorovitch EG, Steinberg RH, Yasumura $\mathrm{S}$, et al. Photoreceptor degeneration in inherited retinal dystrophy delayed by basic fibroblast growth factor. Nolure 1990 ; 347 : 83-6.

4. Lin LF, Mismer D, Lile JD, et al. Purification cloning and expression of ciliary neurotrophic factor (CNTF). Science 1989 ; 246 : 1023-5. 